\title{
Identifying similar transcripts in a related organism from de Bruijn graphs of RNA-Seq data, with applications to the study of salt and waterlogging tolerance in Melilotus
}

\author{
Shuhua Fu' ${ }^{1}$, Peter L. Chang ${ }^{2}$, Maren L. Friesen ${ }^{2,3,4}$, Natasha L. Teakle ${ }^{5,6}$, Aaron M. Tarone ${ }^{7}$ \\ and Sing-Hoi Sze $e^{1,8^{*}}$
}

From 7th IEEE International Conference on Computational Advances in Bio and Medical Sciences (ICCABS 2017)

Orlando, FL, USA. 19-21 October 2017

\begin{abstract}
Background: A popular strategy to study alternative splicing in non-model organisms starts from sequencing the entire transcriptome, then assembling the reads by using de novo transcriptome assembly algorithms to obtain predicted transcripts. A similarity search algorithm is then applied to a related organism to infer possible function of these predicted transcripts. While some of these predictions may be inaccurate and transcripts with low coverage are often missed, we observe that it is possible to obtain a more complete set of transcripts to facilitate possible functional assignments by starting the search from the intermediate de Bruijn graph that contains all branching possibilities.

Results: We develop an algorithm to extract similar transcripts in a related organism by starting the search from the de Bruijn graph that represents the transcriptome instead of from predicted transcripts. We show that our algorithm is able to recover more similar transcripts than existing algorithms, with large improvements in obtaining longer transcripts and a finer resolution of isoforms. We apply our algorithm to study salt and waterlogging tolerance in two Melilotus species by constructing new RNA-Seq libraries.

Conclusions: We have developed an algorithm to identify paths in the de Bruijn graph that correspond to similar transcripts in a related organism directly. Our strategy bypasses the transcript prediction step in RNA-Seq data and makes use of support from evolutionary information.
\end{abstract}

Keywords: de Bruijn graph, RNA-Seq, Melilotus

\section{Background}

As the advance in high-throughput sequencing enables the generation of large volumes of genomic information, it provides researchers the opportunity to study nonmodel organisms even in the absence of a fully sequenced genome. These studies often start from sequencing the entire transcriptome, while additional software is applied

\footnotetext{
*Correspondence: shsze@cse.tamu.edu

'Department of Biochemistry \& Biophysics, Texas A\&M University, College Station 77843 , TX, USA

${ }^{8}$ Department of Computer Science and Engineering, Texas A\&M University, College Station 77843, TX, USA

Full list of author information is available at the end of the article
}

to process the data. An important mechanism to study is alternative splicing, which is crucial to a variety of biological functions. The goal of these studies is to recover as many isoforms as possible in order to understand the underlying biological processes.

In the presence of a reference database, there are two strategies for analyzing transcriptome data. Mapping-first algorithms perform splice-aware alignment of the reads to the reference genome to reconstruct the transcripts $[1,2]$. While these algorithms can construct transcripts independent of known splice sites and identify novel mRNA products, they only allow very few differences during the alignment. Alternatively, when a reference genome

(c) The Author(s). 2019 Open Access This article is distributed under the terms of the Creative Commons Attribution 4.0

International License (http://creativecommons.org/licenses/by/4.0/), which permits unrestricted use, distribution, and reproduction in any medium, provided you give appropriate credit to the original author(s) and the source, provide a link to the Creative Commons license, and indicate if changes were made. The Creative Commons Public Domain Dedication waiver (http://creativecommons.org/publicdomain/zero/1.0/) applies to the data made available in this article, unless otherwise stated. 
is not available but a reference transcriptome is available, transcript quantification algorithms can be applied to analyze differential expression of genes [3, 4].

In the absence of a reference database, an alternative strategy is to employ de novo sequence assembly algorithms [5-12]. A popular strategy of transcriptome assembly algorithms is to assemble the reads by obtaining a de Bruijn graph that represents the transcriptome [12-15].

Although the de Bruijn graph contains all branching possibilities, an additional step is needed to obtain predicted transcripts from the graph. To obtain information about possible function of these predicted transcripts, a similarity search algorithm such as BLAST [16] is then applied to identify similar transcripts in a related organism. In non-model organisms where a fully sequenced genome is not available, this step is the most reliable way to facilitate possible functional assignments. Since the predicted transcripts are constructed based on coverage information, one shortcoming of this approach is that sequences with low coverage are often ignored leading to missed transcripts. The later BLAST step to a related organism then starts from this relatively incomplete set of predicted transcripts.

Instead of performing similarity search from the predicted transcripts, we observe that it is possible to obtain a more complete set of similar transcripts if we start the search from the de Bruijn graph directly (see Fig. 1). This strategy bypasses the transcript prediction step and makes use of support from evolutionary information. Since the graph retains more information from the transcriptome data, transcripts that have low coverage can still be recovered if they have high similarity to the ones from the

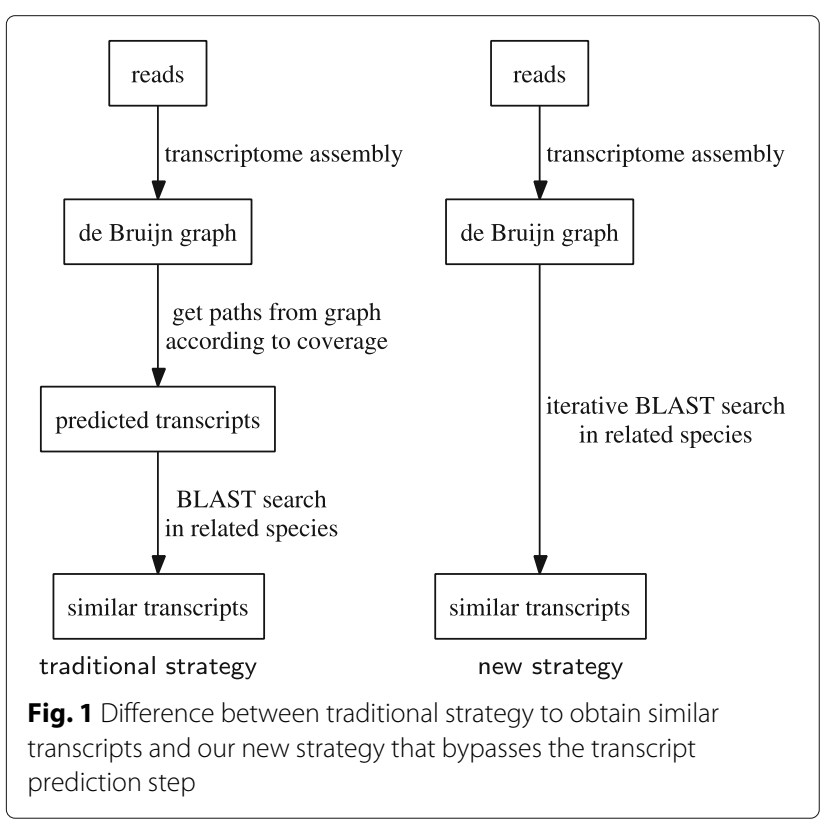

related organism. Wu et al. [17] employed a similar strategy in metagenomics to extract paths directly from the de Bruijn graph that correspond to homologous genes from closely related species. Bao et al. [18] utilized genomic information from the same organism or a related organism (instead of transcripts from a related organism) to improve de novo transcriptome assemblies by first identifying exons from alignments.

While the strategy of applying BLAST from each node in a de Bruijn graph to a related organism can already give a lot of hits, it is possible that some significant hits are missed since the sequence within a node may be too short. There is a need to identify paths in the de Bruijn graph that are similar to transcripts from the related organism. Since the number of possible paths that can be constructed from the de Bruijn graph can be very large, it is not feasible to enumerate all of them.

We develop a heuristic extension algorithm that starts by enumerating short paths in the de Bruijn graph, and iteratively extends these paths in the most promising direction rather than in all possible directions. This procedure generalizes the BLAST algorithm to allow a nonlinear query structure instead of a query sequence. Fu et al. [19] utilized a similar heuristic algorithm to simultaneously extend paths in two de Bruijn graphs in order to compare the transcriptomes of two related organisms at the same time. Zhong et al. [20] employed a genecentric approach in metagenomics to extend an assembly graph structure by identifying reads that are related to assembled protein sequences. Note that our strategy is different from the one in [17] that uses optimal alignment to extend paths due to the smaller scale of metagenomic data. We compare the performance of our algorithm that starts the search from the de Bruijn graph against existing algorithms that employ the strategy of first obtaining predicted transcripts then applying BLAST to obtain similar transcripts.

We validate our algorithm by extracting reads from publicly available RNA-Seq libraries. We construct new RNA-Seq libraries for the non-model organisms Melilotus albus and Melilotus siculus, and apply our algorithm to study salt and waterlogging tolerance in these two species.

\section{Methods}

Given a set of reads and a parameter $k$, a popular strategy of transcriptome assembly algorithms is to assemble these reads into a de Bruijn graph that represents the transcriptome. By taking each $k$-mer that appears within the reads as a vertex, and connecting two $k$-mers by a directed edge if the $(k-1)$-suffix of the first $k$-mer is the same as the $(k-1)$-prefix of the second $k$-mer, the de Bruijn graph implicitly assembles the reads by linking together the same $k$-mer that comes from different reads [21, 22]. 
This strategy is very popular among short read assembly algorithms [6, 7, 9-11].

To minimize the effect of sequencing errors, these algorithms remove short tips and further simplify the de Bruijn graph by collapsing similar paths. Each linear path that contains a sequence of vertices with no branches is collapsed into a single node, and a $k$-mer coverage cutoff $c$ is imposed to remove low coverage nodes [9-11]. We develop an algorithm to identify paths in the de Bruijn graph that correspond to similar transcripts in a related organism. Each extracted path can be considered as a predicted transcript in the original organism.

\section{Initial choice of contigs to extend}

For each transcript in a related organism, our goal is to recover the best path in the de Bruijn graph that corresponds to the transcript. Our approach is based on the seed-extension strategy that starts from short paths, and iteratively extends these paths in the most promising direction. We start the search from nodes in a de Bruijn graph that correspond to contigs from short read assembly algorithms [9-11].

Given a de Bruijn graph, a database of known transcripts in a related organism and an $e$-value cutoff $e_{f}$, we first apply BLAST from each node in the de Bruijn graph to the transcript database to obtain all hits with $e$-value below $e_{i}$, where $e_{i}>e_{f}$. The extra $e$-value cutoff $e_{i}$ is chosen to allow the initial seed nodes to be of lower quality. Some of these nodes can be extended later into longer paths that are of higher quality.

For each transcript in the database, we extract the top $n$ nodes in the de Bruijn graph that give the best BLAST hits to it, where $n$ is a given parameter. The resulting collection of nodes over all transcripts in the database becomes the set of all nodes that our heuristic extension algorithm will start from, which are the ones that are most likely to have correspondences with transcripts in the database. Note that more stringent values of $k$ and the $k$-mer coverage cutoff $c$ can provide longer nodes to start with but can also lead to missed nodes.

\section{Heuristic extension}

For each node $u$ in the collection, we extend its sequence by one node along all outgoing edges from $u$, and apply BLAST from each of these extended sequences to the transcript database (see Fig. 2). If at least one of these extended sequences gives a better $e$-value, we extract the top extended path that gives the best $e$-value. We repeat the extension procedure starting from this new path until either there are no more outgoing edges to extend from or the $e$-value no longer improves.

Note that during each extension, only one best direction is chosen. Extending in more than one direction is very time-consuming since the number of possibilities can be

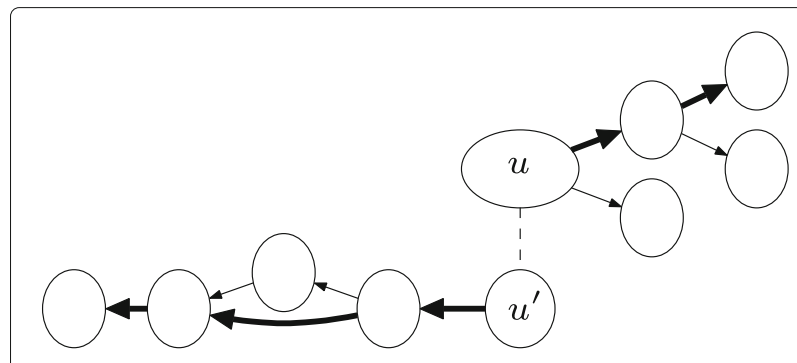

Fig. 2 Illustration of the heuristic extension procedure from a node $u$ and its twin node $u^{\prime}$ until the $e$-value no longer improves (extension proceeds along bold edges)

exponential even in the absence of cycles. Although it is possible that the real best path may be missed, it is still possible to resolve different isoforms since the heuristic extension procedure starts independently from multiple nodes, some of which may be specific to particular isoforms. The procedure can be applied even in the presence of cycles in the de Bruijn graph since the $e$-value cannot improve indefinitely.

We perform a similar procedure on the node $u^{\prime}$ that is the twin node of $u$, which represents the reverse complementary sequence of $k$-mers on the opposite strand, and try to extend it in the opposite direction (see Fig. 2). In addition to adding these two extended paths from $u$ and $u^{\prime}$ to the set of candidate paths, we also merge the twin path that is complementary to the extended path from $u^{\prime}$ with the extended path from $u$ to obtain a longer path. We add the merged path to the set of candidate paths and identify its best BLAST hit in the transcript database.

\section{Extraction of similar transcripts}

At the end of the procedure, for each transcript in the database, we report the top path that gives the best $e$-value to it among all the candidate paths if such a path exists, where the set of candidate paths includes all paths that BLAST has been applied. Only the nodes of a path that are in the best BLAST alignment are reported. It is possible that some of these paths may be the same or very similar for different transcripts in the database.

\section{Melilotus RNA-Seq}

mRNA was extracted from Melilotus albus and Melilotus siculus using a Qiagen Oligotex mRNA mini kit. Fragmentation of mRNA was done using an Ambion fragmentation buffer. Construction of the cDNA library was based on the Illumina protocol. First strand cDNA synthesis was done using Random Hexamer Primers (Invitrogen) and second strand synthesized using a DNA Polymerase 1 (Promega). End repair was carried out to create uniform blunt ends (Epicentre End-IT repair kit). Unique 4 bp adaptors (Illumina) were added so that the libraries could be pooled for 
sequencing. An 'A' base was added using a Klenow enzyme $\left(3^{\prime}\right.$ to $5^{\prime}$ exo minus, NEB) and adaptor ligation was performed using Epicentre Fast-Link DNA ligation kit. The cDNA template was run on a $2 \%$ agarose gel at $120 \mathrm{~V}$ for 60 minutes and fragments of approximately $200-500 \mathrm{bp}$ were removed and purified (Zymo gel purification kit). The purified cDNA template was PCR enriched using the Illumina primers and a Phusion polymerase (NEB). The library was quantified using an Invitrogen Qubit fluorometer. Libraries were sequenced on an Illumina Genome Analyzer II under normal conditions and conditions associated with salt tolerance and/or waterlogging tolerance as single-end $100 \mathrm{bp}$ reads, which were trimmed to $71 \mathrm{bp}$.

\section{Results and discussion}

To assess the performance of our algorithm extContig, we extracted reads from publicly available RNASeq libraries (see Table 1). We validate our algorithm on model organisms by applying BLAST to a database of annotated transcripts in each model organism itself and in two other related model organisms with varying evolutionary distances, including Schizosaccharomyces pombe against another yeast species Saccharomyces cerevisiae and another fungus Neurospora crassa, Drosophila melanogaster against another Drosophila species Drosophila pseudoobscura and mosquito Anopheles gambiae, Homo sapiens against squirrel monkey

Table 1 Data sets used in the evaluation of our heuristic extension algorithm, with organism indicating the starting organism, related organisms indicating the related model organisms that BLAST is applied to, library indicating the total number of libraries, size indicating the total number of bases in all the reads after quality trimming, and reference indicating the publication that describes the libraries

\begin{tabular}{lllll}
\hline Organism & Related organisms & Library & Size & Reference \\
\hline S. pombe & $\begin{array}{l}\text { S. cerevisiae } \\
\text { N. crassa }\end{array}$ & 32 & $17 \mathrm{G}$ & {$[12]$} \\
D. melanogaster & $\begin{array}{l}\text { D. pseudoobscura } \\
\text { A.gambiae }\end{array}$ & 13 & $9.6 \mathrm{G}$ & {$[37]$} \\
& S. boliviensis & 4 & $16 \mathrm{G}$ & {$[38]$} \\
H. sapiens & M. musculus & & & \\
& A. lyrata & 5 & $16 \mathrm{G}$ & {$[39]$} \\
A. thaliana & O. sativa & & & \\
& D. melanogaster & 9 & $4.6 \mathrm{G}$ & {$[23]$} \\
L. sericata & H. sapiens & 13 & $61 \mathrm{G}$ & {$[24]$} \\
H. glaber & H. sapiens & 10 & $66 \mathrm{G}$ & {$[25]$} \\
C. sociabilis & A. thaliana & 3 & $8.6 \mathrm{G}$ & {$[26]$} \\
C. arietinum & A. thaliana & 12 & $5.5 \mathrm{G}$ & New data \\
M. albus & A. thaliana & 12 & $5.4 \mathrm{G}$ & New data \\
M. siculus & & &
\end{tabular}

Saimiri boliviensis and mouse Mus musculus, and Arabidopsis thaliana against another Arabidopsis species Arabidopsis lyrata and rice Oryza sativa.

We evaluate the performance of our algorithm on publicly available RNA-Seq libraries from four non-model organisms. The blow fly Lucilia sericata is important in medicine, forensic science and agriculture due to its filth feeding habits, its use in maggot therapy, its colonization of human and animal remains, and its ability to cause myiasis in vertebrates [23]. The naked mole rat Heterocephalus glaber is important in medicine and in biomedical research due to its resistance to cancer and delayed aging, and its ability to live in adverse conditions [24]. The rodent Ctenomys sociabilis is important in the study of social behavior of mammals and the relationship to gene expression [25]. The chickpea Cicer arietinum is one of the most consumed legume crops that grows in arid areas with low productivity [26]. Similarity search is performed from $L$. sericata to the model organism $D$. melanogaster, from $H$. glaber and C. sociabilis to the model organism $H$. sapiens, and from $C$. arietinum to the model organism $A$. thaliana. The searches that are applied against the same model organism have varying evolutionary distances.

We have constructed new RNA-Seq libraries for the non-model organisms Melilotus albus and Melilotus siculus, which are important in the study of salt and waterlogging tolerance of forage plants [27]. Genomic information on the species will enable the dissection of coumarin production that can be utilized in pharmaceutical development [28]. Similarity search is performed from M. albus and $M$. siculus to the model organism $A$. thaliana.

We trimmed each read by removing all positions including and to the right of the first position that has a quality score of less than 15. For smaller data sets (including D. melanogaster, L. sericata, C. arietinum, M. albus and M. siculus), we compare the performance of our heuristic extension algorithm extVelvet starting from the de Bruijn graph given by Velvet [9] against the performance of Oases [14] that is a postprocessing module of Velvet. Since Oases requires that Velvet is run without coverage cutoff and then applies the coverage cutoff itself, we use the de Bruijn graph within Oases that is modified from Velvet's original de Bruijn graph. For the other larger data sets, we compare the performance of our heuristic extension algorithm extABySS starting from the de Bruijn graph given by ABySS [10] against the performance of Trans-ABySS [13] that is a postprocessing module of ABySS. In each case, we compare the change recovered by Oases and Trans-ABySS to the change recovered by extVelvet and extABySS respectively over the values recovered by their base algorithms Velvet and ABySS respectively.

We applied each algorithm over $k=25,31$, and $c=$ $3,5,10$ for smaller data sets and $c=10,20,50$ for 
larger data sets. BLAST is applied from predicted transcripts in Oases and Trans-ABySS, from paths in the de Bruijn graph in extVelvet and extABySS, and from contigs in Velvet/Oases and ABySS. When comparing each model organism against itself, nucleotide BLAST search is applied to a database of gene transcripts with initial $e$-value cutoff $e_{i}=10^{-15}$ and final $e$-value cutoff $e_{f}=$ $10^{-100}$. In the other cases, translated BLAST search is applied to a database of protein transcripts in a related organism with initial $e$-value cutoff $e_{i}=10^{-6}$ and final $e$-value cutoff $e_{f}=10^{-20}$. For each transcript in the database, the top 8 nodes (and their twin nodes) are chosen to form the initial nodes for extension. Additional criteria are imposed to extend past very short nodes.

\section{Transcript recovery}

We assess the performance of each algorithm in recovering transcripts by investigating the amount of similar transcripts obtained and the amount of recovered transcripts that are close to full length. While the performance depends on the size of RNA-Seq data, the complexity of transcriptomes, the evolutionary distance between organisms and the assembly algorithm that is being used, Fig. 3 shows that Oases and Trans-ABySS recover more similar transcripts than their base algorithms Velvet and ABySS, while extVelvet and extABySS recover even more. The improvement of Trans-ABySS is small when compared to ABySS, which leads to a much larger improvement of extABySS over TransABySS. These improvements are not absolute since different algorithms can recover different sets of similar transcripts.

Figure 4 shows that extVelvet and extABySS can recover more similar transcripts that are close to full length than Oases and Trans-ABySS in most cases. Both Oases and extVelvet (or Trans-ABySS and extABySS) can recover more full length transcripts than Velvet (or ABySS), which can be a few times more in some cases.

\section{Alternative splicing}

We assess the ability of each algorithm in distinguishing between isoforms by considering exons in genes with multiple isoforms. Figure 5 shows that extVelvet and extABySS are able to recover a larger number of such exons in most cases.

Figure 6 shows examples in which extVelvet and extABySS can better resolve isoforms with respect to a related organism, including the $Z D H H C 16$ gene, which is a zinc finger protein that may be involved in apoptosis regulation [29]; the $d S a r m$ gene, in which the loss of its function protects against injury-induced axon death [30]; the STAT3 gene, which is an acute-phase response factor in which the isoforms have unique functions [31]; and the AT4G34660 gene, which is a SH3 domain- containing protein that is involved in clathrin-mediated vesicle trafficking [32].

\section{Translocated transcripts}

We assess the reliability of each algorithm by identifying the amount of translocated transcripts that are returned. As reported by GMAP [33], Fig. 7 shows that extVelvet and extABySS recover a larger number of similar transcripts that are uniquely mapped than Oases and TransABySS, with extVelvet returning less translocated transcripts than Oases when the starting organism is different from the related organism, and extABySS returning a few times more translocated transcripts than Trans-ABySS in most cases (except for $A$. thaliana when Trans-ABySS returns very few translocated transcripts).

\section{Gene expression}

We assess the ability of each algorithm in recovering transcripts at different expression levels. We apply eXpress [4] to the reads in each data set with respect to the database of recovered similar transcripts in the starting organism that are close to full length to obtain FPKM expression estimates. Figure 8 shows that extABySS is able to recover a higher proportion of full length transcripts with low coverage than ABySS and Trans-ABySS.

\section{Melilotus albus and Melilotus siculus}

In order to study salt and waterlogging tolerance of the two Melilotus species, we apply our algorithm extVelvet starting from each species both to the model organism A. thaliana and to the non-model organism Medicago truncatula. Although M. truncatula is not as well annotated as A. thaliana, it is closer in evolutionary distance to Melilotus and will give better results. We assess the differences between the two species by applying GO Term Finder [34] to the two sets of genes that are present in recovered similar transcripts from M. albus and M. siculus when our algorithm is applied to A. thaliana and $M$. truncatula, and identify significant GO terms with Bonferroni corrected $p$-value below 0.01 within the biological process ontology. Figures 9 and 10 show that while a large number of genes in recovered similar transcripts and significant GO terms are shared by the two species, a small number of results that are unique to each species can be found (see Additional file 1 for details). For $M$. albus, the most notable unique genes are related to RNA splicing, response to brassinosteroid stimulus, and developmental regulation. For M. siculus, the most notable unique genes are related to response to karrikin (a smoke-derived molecule that regulates seed development), nucleic acid metabolism, negative regulation of cell differentiation, and nucleus organization. These results suggest large differences in gene expression strategies of these species, as they respond to the same stressful environments. 


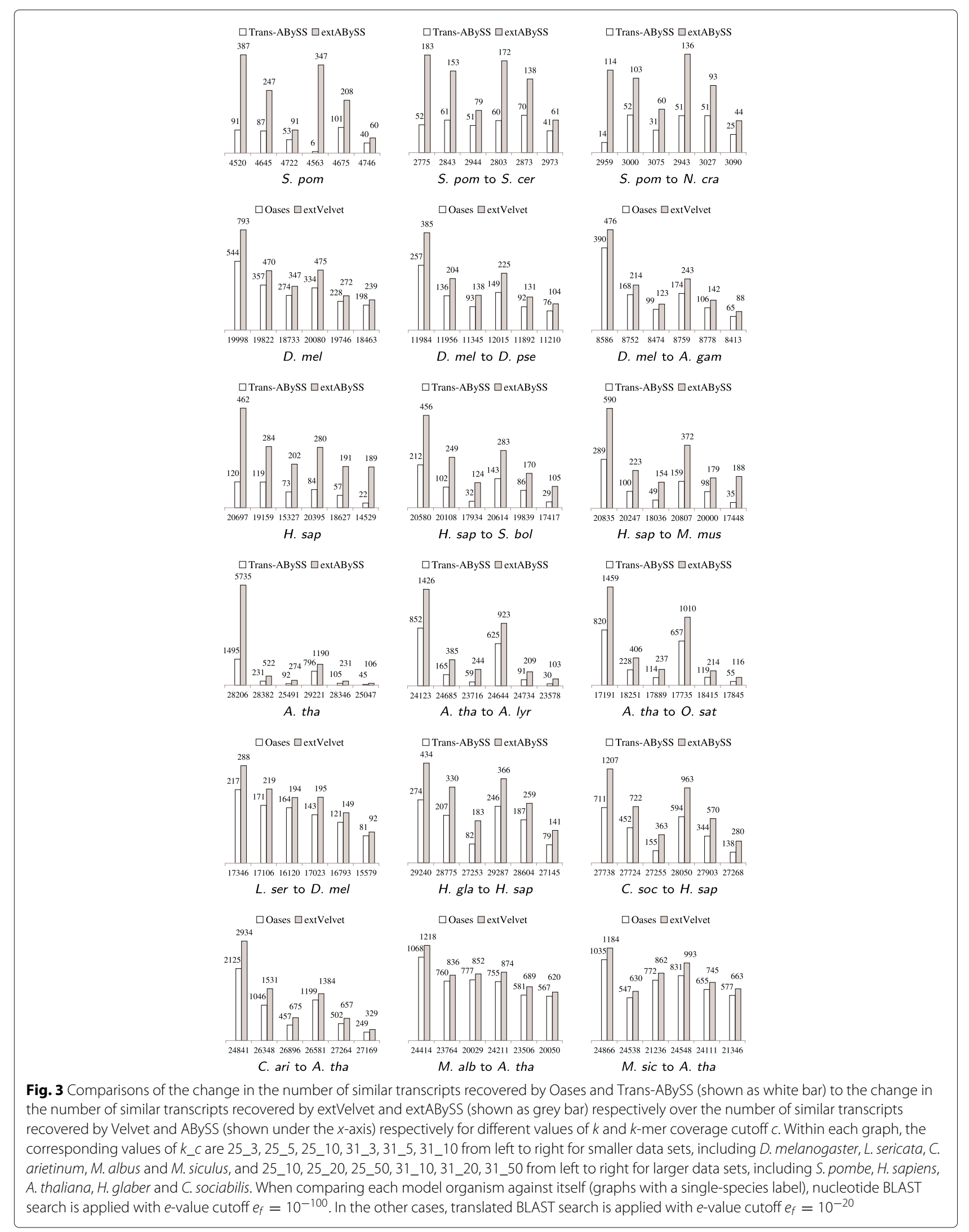



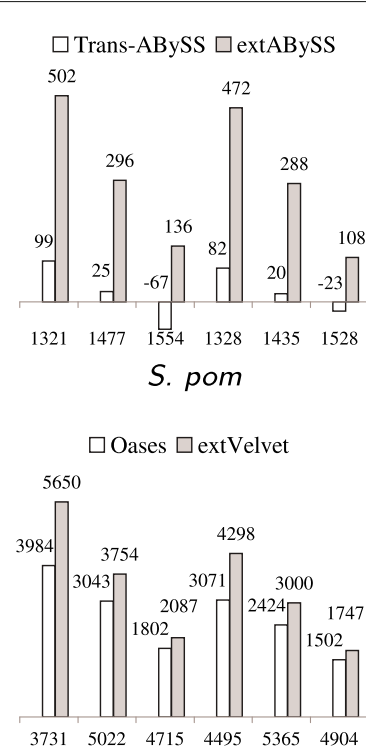

D. mel

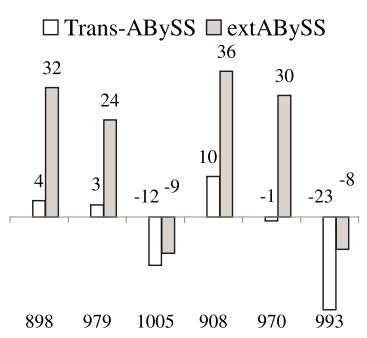

S. pom to $S$. cer

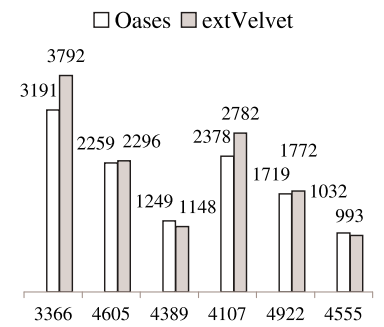

D. mel to $D$. pse

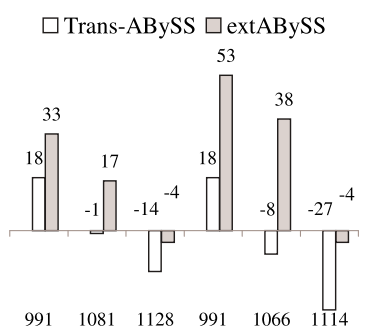

S. pom to N. cra

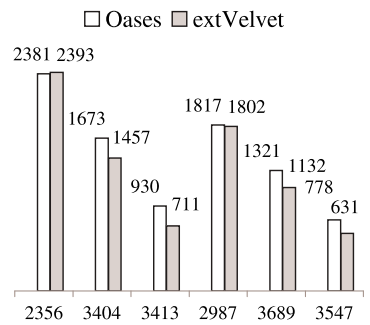

D. mel to $A$. gam $\square$ Trans-ABySS $\square$ extABySS

$$
1109
$$

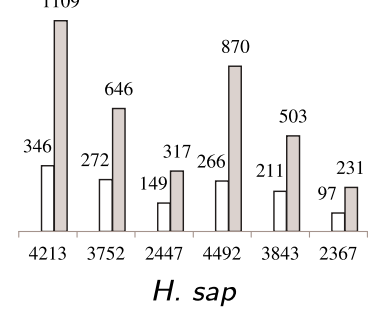

$\square$ Trans-ABySS $\square$ extABySS 9531

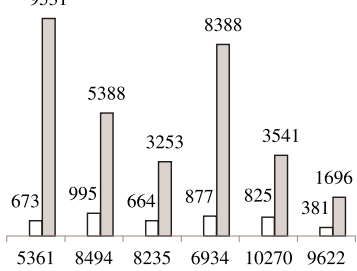

A. tha

\section{$\square$ Trans-ABySS $\square$ extABySS} 703

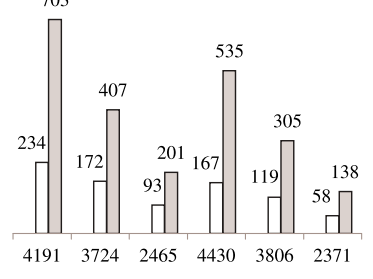

H. sap to $S$. bol

$$
\begin{aligned}
& \square \text { Trans-ABySS } \square \text { extABySS } \\
& 7065
\end{aligned}
$$$$
\underset{5095}{7}
$$

A. tha to A. lyr

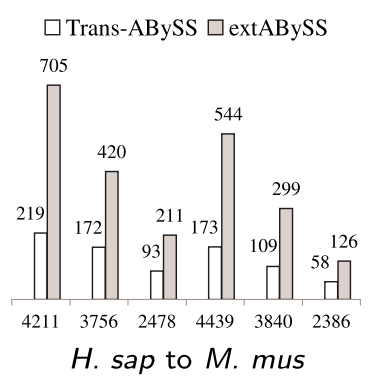

$\square$ Trans-ABySS $\square$ extABySS 3276

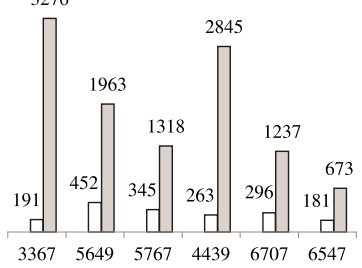

A. tha to $O$. sat

Fig. 4 Comparisons of the change in the number of similar transcripts in the starting organism that are $80 \%$ full length transcripts (100\% full length transcripts when S. pombe is the starting organism) and recovered by Oases and Trans-ABySS to the change in the ones recovered by extVelvet and extABySS respectively over the ones recovered by Velvet and ABySS respectively for different values of $k$ and $k$-mer coverage cutoff $c$. Notations are the same as in Figure 3. These transcripts are the ones in which $80 \%$ (100\% when S. pombe is the starting organism) of the coding region is included in the best BLAST alignment

To assess gene expression under different conditions, we apply edgeR [35] on the FPKM expression estimates given by eXpress [4] to obtain a set of differentially expressed genes under one condition against another condition with $q$-value below 0.01 , and apply GO Term Finder [34] to identify significant GO terms within each set of genes.

Tables 2 and 3 show that differentially expressed genes can be identified in all cases, with some of them associated with significant GO terms (see Additional file 1 for details). In the results from libraries associated with salt and waterlogging tolerance against control, many genes are found to be differentially expressed in $M$. albus that are related to response to chemical stimulus, stress, organic substance, inorganic substance, abiotic stimulus, and oxygen stress. There is also a significant enrichment of genes that respond to hormones, with at least one of these genes indicating ethylene physiology as important in the stress response. In contrast, very few genes 

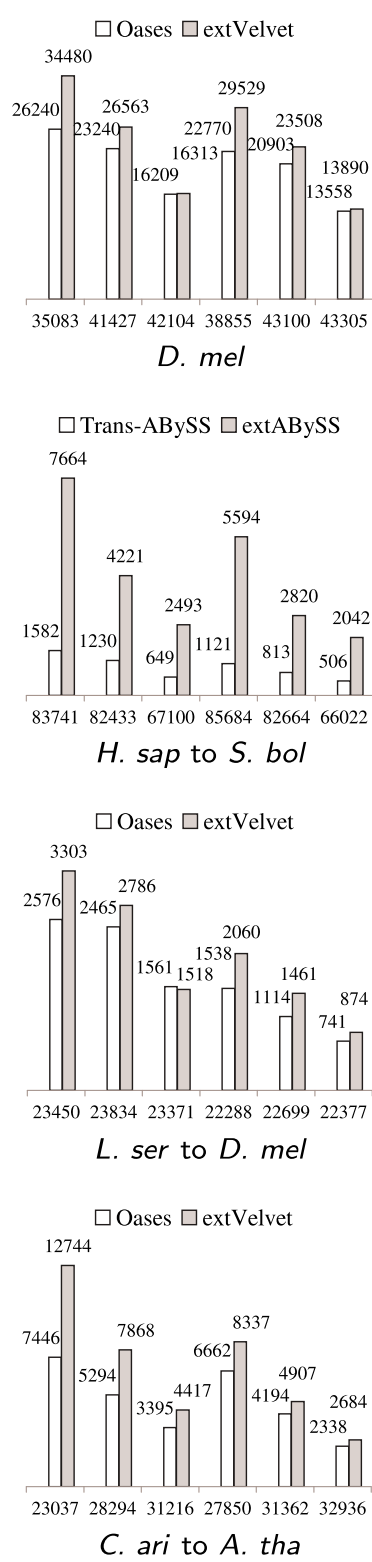
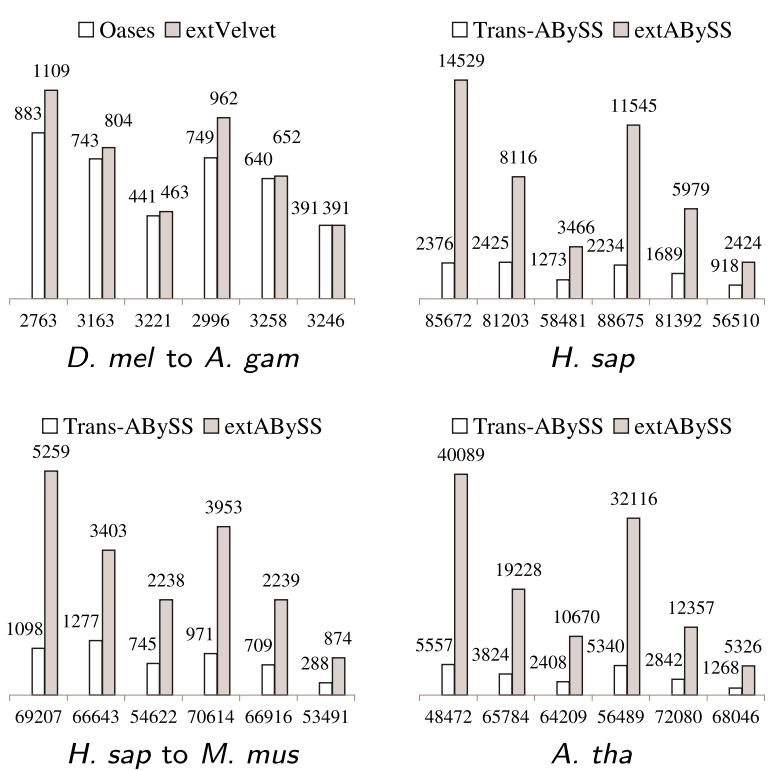

A. tha
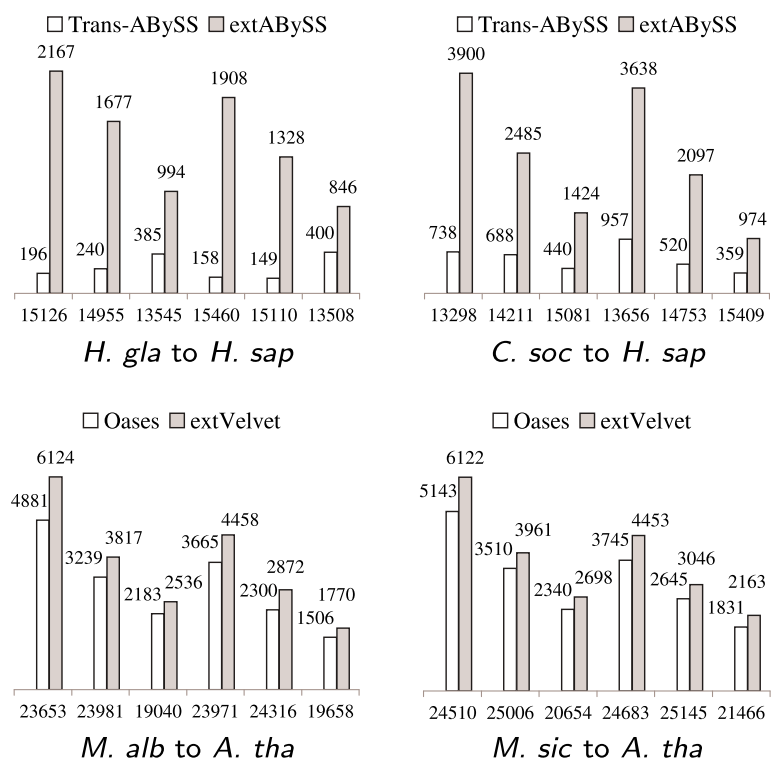

Fig. 5 Comparisons of the change in the total number of exons in genes with multiple isoforms recovered by Oases and Trans-ABySS to the change in the ones recovered by extVelvet and extABySS respectively over the ones recovered by Velvet and ABySS respectively for different values of $k$ and $k$-mer coverage cutoff $c$. Notations are the same as in Figure 3. Exons within isoforms that do not have the same starting position or the same ending position are considered to be distinct. An exon is recovered if it has some overlap with the best BLAST alignment. Exons within mRNAs are considered when comparing each model organism against itself, while exons within coding regions of the related model organism are considered in the other cases. Results for S. pombe are not included since there is little alternative splicing, while a few other results are not included due to poor annotations of alternative splicing in the related model organisms

are found to be differentially expressed in M. siculus. Among these genes, chalcone-flavanone, terpenoid, and ferulic acid physiology are implicated in the biology of the stress response. These results provide further basis to study the genes that are responsible for the major differences in salt and waterlogging tolerance of the two species.

\section{Conclusions}

Since the main memory requirement of our algorithm is for storing the de Bruijn graph and performing BLAST searches, our heuristic extension algorithms extVelvet and extABySS are much less memory intensive and more easily parallelizable than the base algorithms Velvet and ABySS [36]. Since a postprocessing module such as Oases 

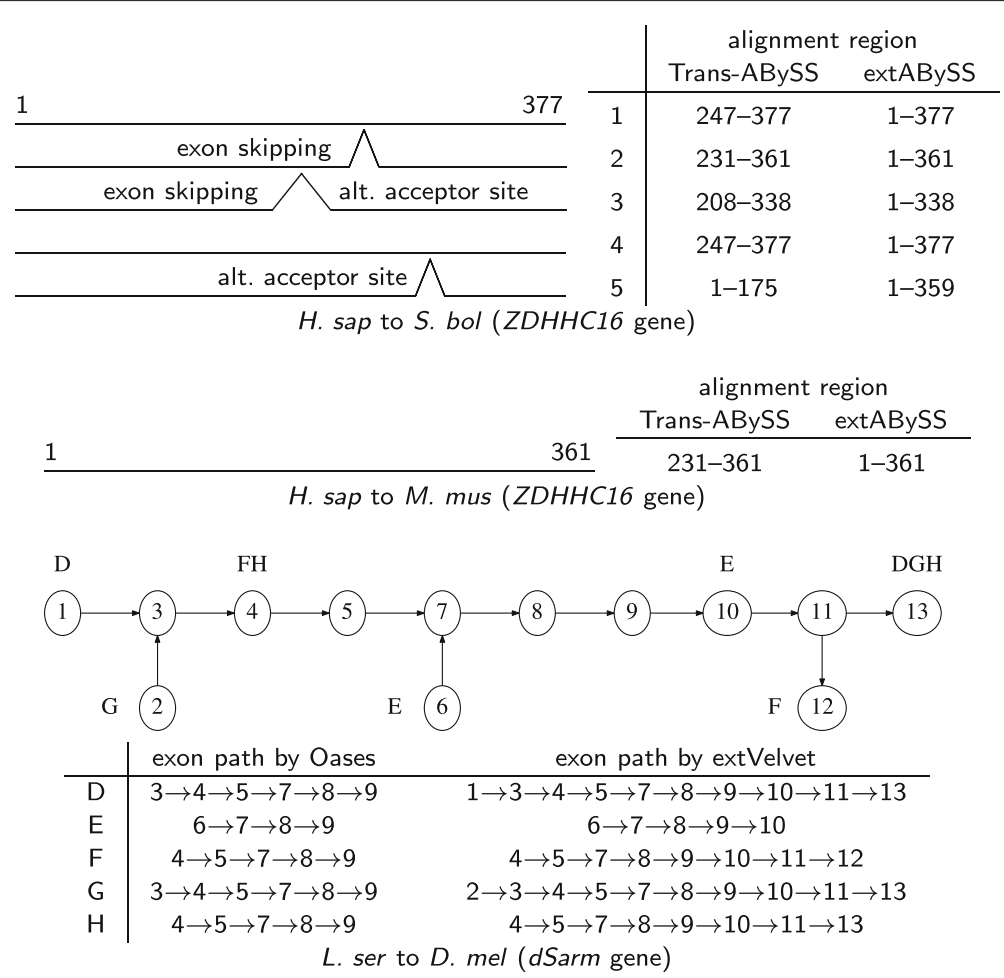

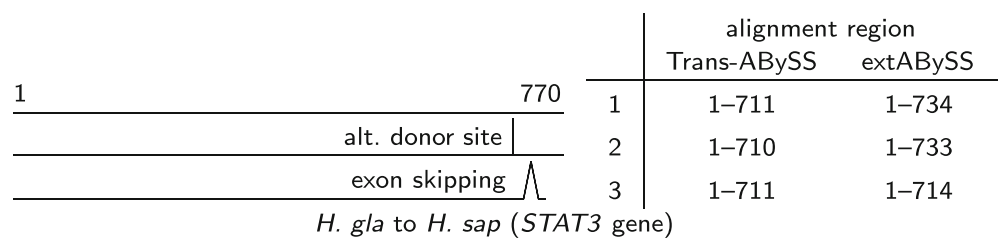

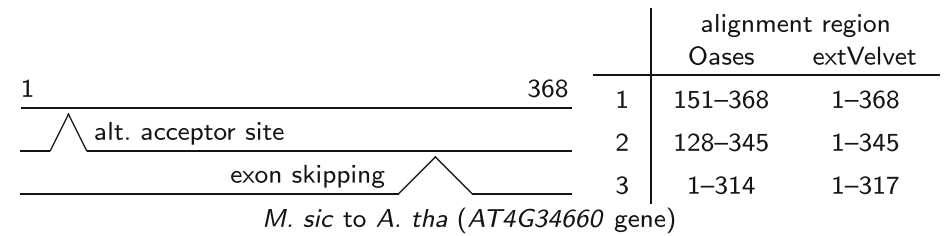

Fig. 6 Examples of the resolution of alternative splicing with respect to a related organism. The splicing structures are on exons in the coding region of the related organism. For the $d S$ arm gene, uppercase letters indicate isoforms and their start/end exons, with Oases resolving less isoforms than extVelvet. In the other splicing structures, the isoforms are drawn to scale and the starting and ending amino acid positions of isoform 1 are shown. For the ZDHHC16 gene, Trans-ABySS cannot resolve its different isoforms on S. boliviensis, and recovers a shorter segment of it on M. musculus with no known alternative splicing. Trans-ABySS cannot resolve isoforms 1 and 3 of the STAT3 gene, while Oases cannot resolve isoforms 1 and 2 of the AT4G34660 gene

may need more memory than its base algorithm Velvet, our heuristic extension algorithm provides an alternative in these cases. Iterative BLAST searches can be performed independently in parallel by assigning disjoint subsets of nodes to different processors.

The running time of our algorithm has large dependence on the number of nodes that are chosen for extension (see Table 4). This in turn depends on the size of RNA-Seq data and the complexity of transcriptomes, which are reflected by the number of nodes in the de Bruijn graph and the number of transcripts in the database. It also depends on the evolutionary distance between the starting organism and the related model organism. As the evolutionary distance increases, both the number of nodes that are chosen for extension and the running time decrease. When applying to a different related organism, our running time in terms of processorhours is at most a few to 10 times more than the base algorithm in almost all cases, and it can be much less in some cases. 


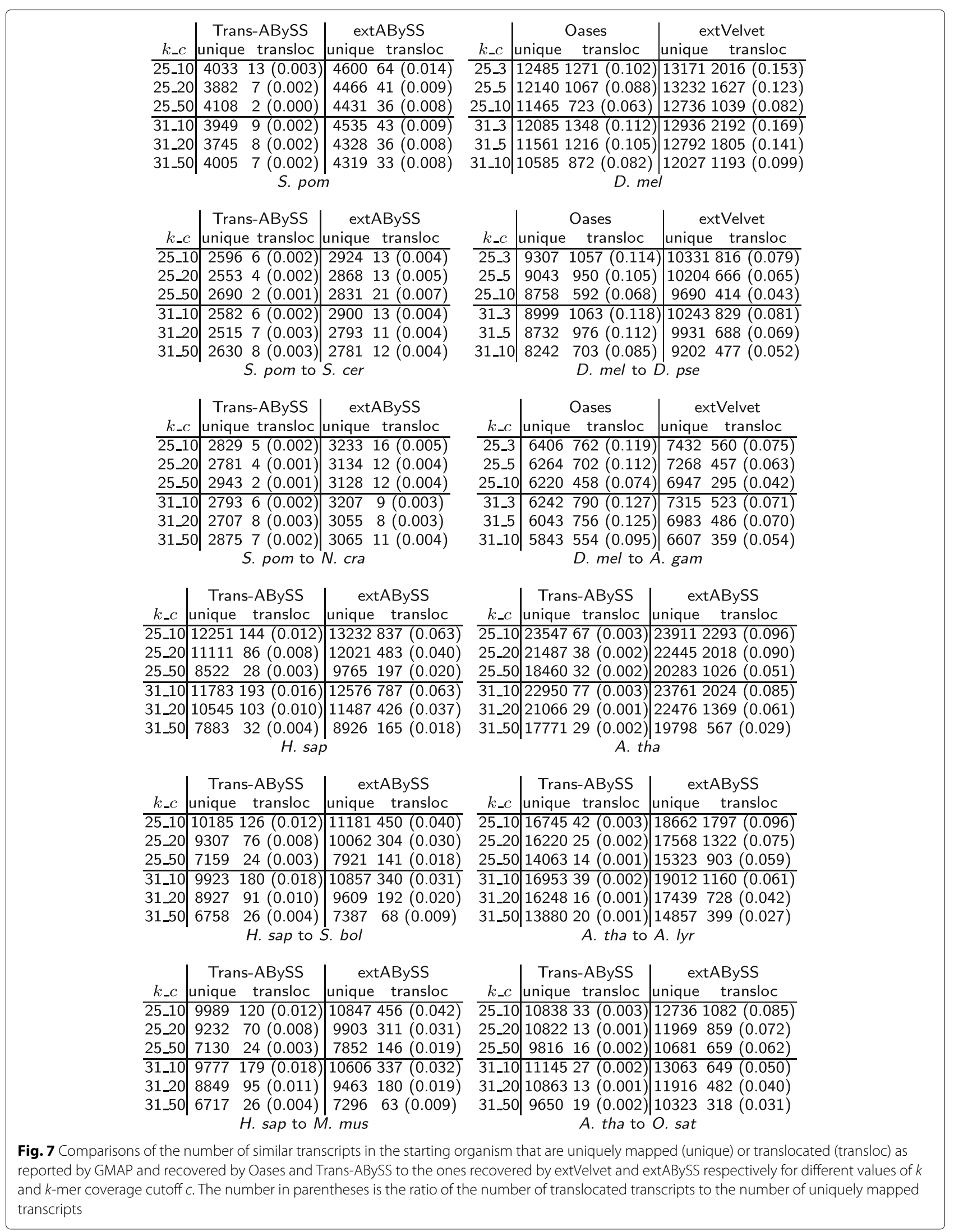



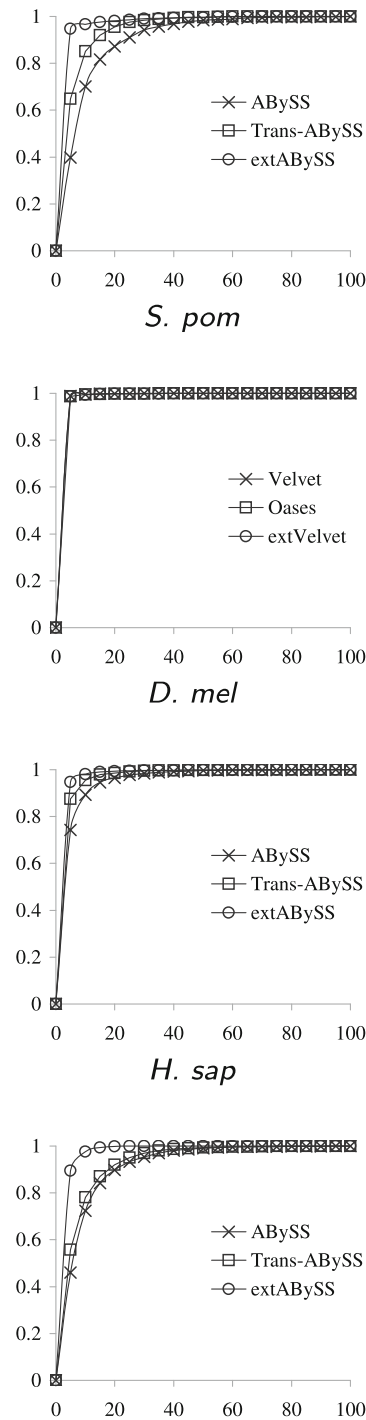

A. tha

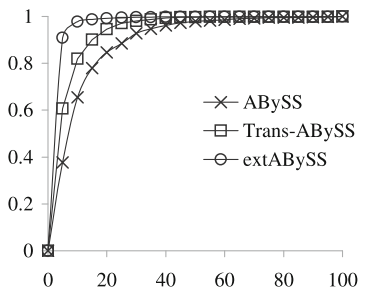

S. pom to $S$. cer
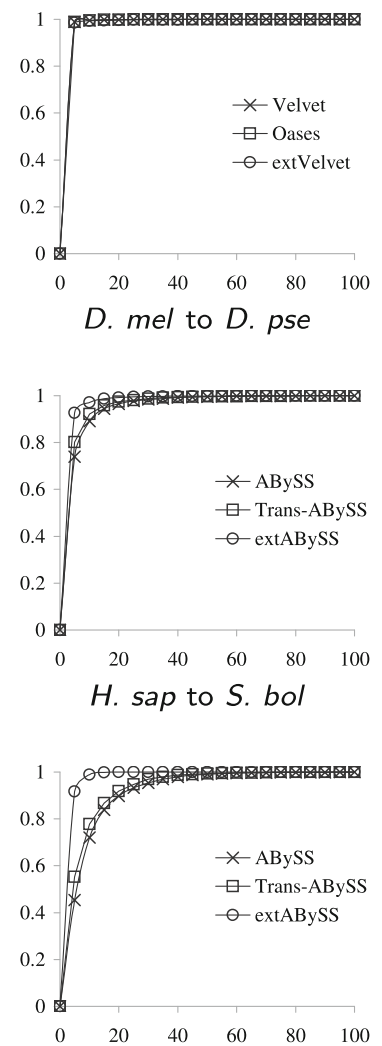

A. tha to $A$. lyr

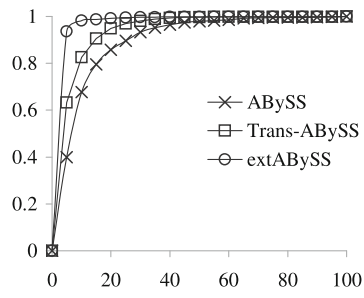

S. pom to N. cra
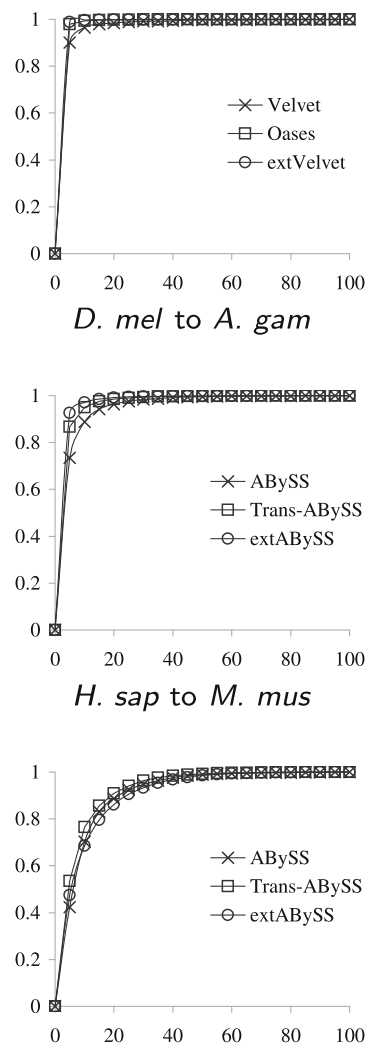

A. tha to $O$. sat

Fig. 8 Comparisons of the cumulative distribution of the FPKM expression estimates of similar transcripts in the starting organism that are $80 \%$ full length transcripts (100\% full length transcripts when S. pombe is the starting organism) and recovered by Velvet, Oases and extVelvet (or by ABySS, Trans-ABySS and extABySS), with the range of FPKM values in each assembly divided into 20 intervals of equal width and shown as a percentage under the $x$-axis. The least stringent values of $k_{-} c$ are used in each case, which is 25_3 for D. melanogaster and 25_10 for the other organisms

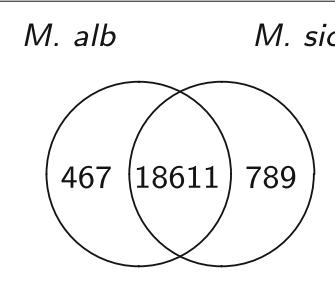

to $A$. tha

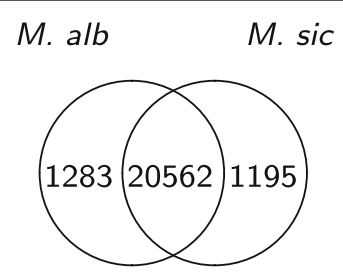

to M. tru

Fig. 9 Venn diagrams of the number of genes that are present in recovered similar transcripts from $M$. albus and $M$. siculus when our algorithm is applied to A. thaliana and M. truncatula in the 25_3 assembly

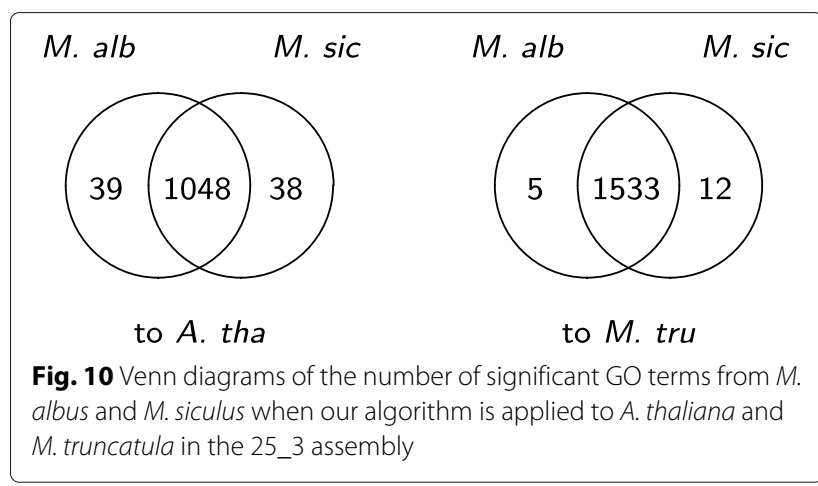


Table 2 Number of differentially expressed genes recovered from M. albus and M. siculus when our algorithm is applied to $A$. thaliana and $M$. truncatula from libraries associated with one condition versus another condition in the 25_3 assembly, with organism indicating the starting organism and its related organism, SvsC indicating salt tolerance versus control, WvsC indicating waterlogging tolerance versus control, SWvsC indicating salt and waterlogging tolerance versus control, SWvsS indicating salt and waterlogging tolerance versus salt tolerance, and SWvsW indicating salt and waterlogging tolerance versus waterlogging tolerance

\begin{tabular}{llllll}
\hline Organism & SvsC & WvsC & SWvsC & SWvsS & SWvsW \\
\hline M. alb to A. tha & 8 & 141 & 81 & 47 & 12 \\
M. sic to A. tha & 39 & 7 & 10 & 45 & 8 \\
M. alb to M. tru & 11 & 220 & 114 & 86 & 17 \\
M. sic to M. tru & 74 & 24 & 31 & 84 & 12 \\
\hline
\end{tabular}

The situation is different in model organisms when similarity searches are performed to the organism itself. Since the BLAST hits are of much higher quality, path extensions can be very time-consuming. In such cases, mapping-first algorithms such as Cufflinks [2] or Scripture [1] could be used instead, which often have better performance since our need to impose a $k$-mer coverage cutoff to simplify the de Bruijn graph for heuristic extension often leads to missed transcripts.

Our heuristic extension strategy cannot be applied to all transcriptome assembly algorithms. On algorithms such as Trinity [12] that first clusters the data and constructs a de Bruijn graph individually for each cluster, each of these graphs has simple structures. Performing heuristic extension on top of these graphs will not lead to significant improvements.

While our strategy cannot replace transcript predictions in de novo assemblies when the goal is to identify novel transcripts that have no similarity to other organisms, we have shown that our strategy can recover more and longer transcripts and can better resolve isoforms when similar transcripts are available from a related organism. The sequence similarity support from the BLAST alignments ensures that the correspondences between the transcripts in the original organism and in the related organism are real.

Table 3 Number of significant GO terms recovered from M. albus and $M$. siculus when our algorithm is applied to A. thaliana and $M$. truncatula from libraries associated with one condition versus another condition in the 25_3 assembly. Notations are the same as in Table 2

\begin{tabular}{llllll}
\hline Organism & SvsC & WvsC & SWvsC & SWvsS & SWvsW \\
\hline M. alb to A. tha & 0 & 23 & 42 & 7 & 0 \\
M. sic to A. tha & 9 & 0 & 0 & 2 & 0 \\
M. alb to M. tru & 2 & 0 & 1 & 0 & 0 \\
M. sic to M. tru & 0 & 0 & 0 & 0 & 0 \\
\hline
\end{tabular}

Table 4 Running time in processor-hours, with the values to the left and to the right of " + " indicating the running time of Velvet and Oases respectively (or ABySS and Trans-ABySS respectively), organism indicating the related model organism, time indicating the running time of extVelvet (or extABySS), chosen indicating the number of nodes that are chosen for extension, de Bruijn indicating the number of nodes in the de Bruijn graph, and database indicating the number of transcripts in the database

\begin{tabular}{|c|c|c|c|c|c|}
\hline Least stringent $k \_c$ & Organism & Time & Chosen & De Bruijn & Database \\
\hline \multirow[t]{3}{*}{ S.pom (84+0.2) } & S.pom & 45 & 41692 & 536894 & 5011 \\
\hline & S.cer & 12 & 15252 & 536894 & 5907 \\
\hline & N. cra & 12 & 16366 & 536894 & 10082 \\
\hline \multirow[t]{3}{*}{ D. mel $(6.7+4.4)$} & D. mel & 238 & 138972 & 459644 & 22102 \\
\hline & D.pse & 67 & 64012 & 459644 & 16071 \\
\hline & A.gam & 32 & 41580 & 459644 & 12659 \\
\hline \multirow[t]{3}{*}{ H. sap $(45+0.2)$} & H. sap & 595 & 222244 & 1133368 & 32787 \\
\hline & S.bol & 490 & 88340 & 1133368 & 25621 \\
\hline & M. mus & 167 & 85166 & 1133368 & 29617 \\
\hline \multirow[t]{3}{*}{ A. tha $(112+0.2)$} & A. tha & 2495 & 423410 & 3111862 & 41671 \\
\hline & A. lyr & 944 & 218760 & 3111862 & 32549 \\
\hline & O. sat & 616 & 144058 & 3111862 & 26777 \\
\hline L. $\operatorname{ser}(1.2+0.2)$ & D. mel & 67 & 41872 & 257700 & 22102 \\
\hline H. gla $(368+0.2)$ & H. sap & 1920 & 192772 & 5457968 & 32799 \\
\hline C. $\operatorname{soc}(440+0.2)$ & H. sap & 1344 & 175690 & 5030586 & 32799 \\
\hline C. $\operatorname{ari}(4.2+4.6)$ & A. tha & 200 & 103524 & 1205362 & 41671 \\
\hline M. alb $(5.8+2.9)$ & A. tha & 79 & 82996 & 562210 & 41671 \\
\hline M. sic $(9.3+6.8)$ & A. tha & 67 & 83718 & 482826 & 41671 \\
\hline
\end{tabular}

\section{Additional file}

Additional file 1: Lists of unique genes that are present in recovered similar transcripts and differentially expressed genes from libraries associated with one condition versus another condition along with significant $\mathrm{GO}$ terms recovered from $M$. albus and $M$. siculus when our algorithm is applied to $A$. thaliana and $M$. truncatula in the $25 \_3$ assembly. (ZIP $101 \mathrm{~kb})$

\section{Acknowledgements}

We thank the reviewers for comments that significantly improve the paper. Computations were performed on the Brazos Cluster and the Whole Systems Genomics Initiative Cluster at Texas A\&M University.

\section{Funding}

This work was supported in part by the National Science Foundation [DBI-0820846, MCB-0951120]; and the National Institute of Justice [2012-DN-BX-K024]. NLT is supported by the Centre for Ecohydrology, a Western Australian State Government Centre of Excellence, and the Australian Government Science and Innovation Award. AMT is supported by start-up funds from the College of Agriculture and Life Sciences at Texas A\&M University and Texas AgriLife Research. Points of view in this document are those of the authors and do not necessarily represent the official position or policies of the U.S. Department of Justice. Publication costs for this work were funded by the Open Access to Knowledge (OAK) Fund at the Texas A\&M University Libraries. 


\section{Availability of data and materials}

The extContig software that implements the algorithm is available at http://faculty.cse.tamu.edu/shsze/extcontig. The newly constructed Melilotus RNA-Seq libraries are available at the Sequence Read Archive (SRP187991, SRP188004)

\section{About this supplement}

This article has been published as part of BMC Genomics Volume 20 Supplement 5, 2019: Selected articles from the 7th IEEE International Conference on Computational Advances in Bio and Medical Sciences (ICCABS 2017): genomics. The full contents of the supplement are available online at https://bmcgenomics.biomedcentral.com/articles/supplements/volume-20supplement-5.

\section{Authors' contributions}

SF, AMT and S-HS designed the computational work. PLC, MLF and NLT performed the molecular experiments. All authors read and approved the final manuscript.

\section{Ethics approval and consent to participate}

Not applicable.

\section{Consent for publication}

Not applicable.

\section{Competing interests}

The authors declare that they have no competing interests.

\section{Publisher's Note}

Springer Nature remains neutral with regard to jurisdictional claims in published maps and institutional affiliations.

\section{Author details}

${ }^{1}$ Department of Biochemistry \& Biophysics, Texas A\&M University, College Station 77843, TX, USA. ${ }^{2}$ Molecular and Computational Biology Section, Department of Biological Sciences, University of Southern California, Los Angeles 90089, CA, USA. ${ }^{3}$ Department of Crop and Soil Sciences, Washington State University, Pullman 99164, WA, USA. ${ }^{4}$ Department of Plant Pathology, Washington State University, Pullman 99164, WA, USA. ${ }^{5}$ Centre for Ecohydrology, The University of Western Australia, 35 Stirling Highway, 6009 Crawley, WA, Australia. ${ }^{6}$ School of Plant Biology (M084), Faculty of Natural and Agricultural Sciences, The University of Western Australia, 35 Stirling Highway, 6009 Crawley, WA Australia. ${ }^{7}$ Department of Entomology, Texas A\&M University, College Station, TX 77843, USA. ${ }^{8}$ Department of Computer Science and Engineering, Texas A\&M University, College Station 77843, TX, USA.

\section{Published: 6 June 2019}

\section{References}

1. Guttman M, Garber M, Levin JZ, Donaghey J, Robinson J, Adiconis X, Fan L, Koziol MJ, Gnirke A, Nusbaum C, Rinn JL, Lander ES, Regev A. Ab initio reconstruction of cell type-specific transcriptomes in mouse reveals the conserved multi-exonic structure of lincRNAs. Nat Biotechnol. 2010;28:503-10.

2. Trapnell C, Williams BA, Pertea G, Mortazavi A, Kwan G, van Baren MJ, Salzberg SL, Wold BJ, Pachter L. Transcript assembly and quantification by RNA-Seq reveals unannotated transcripts and isoform switching during cell differentiation. Nat Biotechnol. 2010;28:511-5.

3. Li B, Dewey CN. RSEM: accurate transcript quantification from RNA-Seq data with or without a reference genome. BMC Bioinformatics. 2011;12: 323.

4. Roberts A, Pachter L. Streaming fragment assignment for real-time analysis of sequencing experiments. Nat Methods. 2013;10:71-3.

5. Dohm JC, Lottaz C, Borodina T, Himmelbauer H. SHARCGS, a fast and highly accurate short-read assembly algorithm for de novo genomic sequencing. Genome Res. 2007;17:1697-706.

6. Butler J, MacCallum I, Kleber M, Shlyakhter IA, Belmonte MK, Lander ES, Nusbaum C, Jaffe DB. ALLPATHS: de novo assembly of whole-genome shotgun microreads. Genome Res. 2008;18:810-20.

7. Chaisson MJ, Pevzner PA. Short read fragment assembly of bacterial genomes. Genome Res. 2008;18:324-30.
8. Hernandez D, François P, Farinelli L, Østerås M, Schrenzel J. de novo bacterial genome sequencing: millions of very short reads assembled on a desktop computer. Genome Res. 2008;18:802-9.

9. Zerbino DR, Birney E. Velvet: algorithms for de novo short read assembly using de Bruijn graphs. Genome Res. 2008;18:821-9.

10. Birol I, Jackman SD, Nielsen CB, Qian JQ, Varhol R, Stazyk G, Morin RD, Zhao Y, Hirst M, Schein JE, Horsman DE, Connors JM, Gascoyne RD, Marra MA, Jones SJM. de novo transcriptome assembly with ABySS Bioinformatics. 2009;25:2872-7.

11. LiR, Zhu H, Ruan J, Qian W, Fang X, Shi Z, Li Y, Li S, Shan G, Kristiansen K, Li S, Yang H, Wang J, Wang J. de novo assembly of human genomes with massively parallel short read sequencing. Genome Res. 2010;20: 265-72.

12. Grabherr MG, Haas BJ, Yassour M, Levin JZ, Thompson DA, Amit I, Adiconis X, Fan L, Raychowdhury R, Zeng Q, Chen Z, Mauceli E, Hacohen N, Gnirke A, Rhind N, di Palma F, Birren BW, Nusbaum C, Lindblad-Toh K, Friedman N, Regev A. Full-length transcriptome assembly from RNA-Seq data without a reference genome. Nat Biotechnol. 2011;29:644-52.

13. Robertson $G$, Schein J, Chiu R, Corbett R, Field M, Jackman SD, Mungall $K$ Lee S, Okada HM, Qian JQ, Griffith M, Raymond A, Thiessen N, Cezard T, Butterfield YS, Newsome R, Chan SK, She R, Varhol R, Kamoh B, Prabhu A-L, Tam A, Zhao Y, Moore RA, Hirst M, Marra MA, Jones SJM, Hoodless PA, Birol I. de novo assembly and analysis of RNA-seq data. Nat Methods. 2010;7:909-12.

14. Schulz MH, Zerbino DR, Vingron M, Birney E. Oases: robust de novo RNA-seq assembly across the dynamic range of expression levels. Bioinformatics. 2012;28:1086-92.

15. Xie Y, Wu G, Tang J, Luo R, Patterson J, Liu S, Huang W, He G, Gu S, Li S, Zhou X, Lam T-W, Li Y, Xu X, Wong GK-S, Wang J. SOAPdenovo-Trans: de novo transcriptome assembly with short RNA-Seq reads. Bioinformatics. 2014;30:1660-6.

16. Altschul SF, Gish W, Miller W, Myers EW, Lipman DJ. Basic local alignment search tool. J Mol Biol. 1990;215:403-10.

17. Wu Y-W, Rho M, Doak TG, Ye Y. Stitching gene fragments with a network matching algorithm improves gene assembly for metagenomics. Bioinformatics. 2012;28:363-9.

18. Bao E, Jiang T, Girke T. BRANCH: boosting RNA-Seq assemblies with partial or related genomic sequences. Bioinformatics. 2013;29:1250-9.

19. Fu S, Tarone AM, Sze S-H. Heuristic pairwise alignment of de Bruijn graphs to facilitate simultaneous transcript discovery in related organisms from RNA-Seq data. BMC Genomics. 2015;16(Suppl 11):5.

20. Zhong C, Yang Y, Yooseph S. GRASP2: fast and memory-efficient gene-centric assembly and homolog search. In: Proceedings of the 7th IEEE International Conference on Computational Advances in Bio and Medical Sciences. IEEE Xplore Digital Library; 2017.

21. Pevzner PA. I-tuple DNA sequencing: computer analysis. J Biomol Struct Dyn. 1989;7:63-73.

22. Idury RM, Waterman MS. A new algorithm for DNA sequence assembly. J Comput Biol. 1995;2:291-306.

23. Sze S-H, Dunham JP, Carey B, Chang PL, Li F, Edman RM, Fjeldsted C, Scott MJ, Nuzhdin SV, Tarone AM. A de novo transcriptome assembly of Lucilia sericata (Diptera: Calliphoridae) with predicted alternative splices, single nucleotide polymorphisms, and transcript expression estimates. Insect Mol Biol. 2012;21:205-21.

24. Kim EB, Fang X, Fushan AA, Huang Z, Lobanov AV, Han L, Marino SM, Sun $X$, Turanov AA, Yang P, Yim SH, Zhao X, Kasaikina MV, Stoletzki N, Peng C, Polak P, Xiong Z, Kiezun A, Zhu Y, Chen Y, Kryukov GV, Zhang Q, Peshkin L, Yang L, Bronson RT, Buffenstein R, Wang B, Han C, Li Q, Chen L, Zhao W, Sunyaev SR, Park TJ, Zhang G, Wang J, Gladyshev VN. Genome sequencing reveals insights into physiology and longevity of the naked mole rat. Nature. 2011;479:223-7.

25. MacManes MD, Lacey EA. The social brain: transcriptome assembly and characterization of the hippocampus from a social subterranean rodent, the colonial tuco-tuco (Ctenomys sociabilis). PLoS ONE. 2012;7:45524.

26. Garg R, Patel RK, Tyagi AK, Jain M. de novo assembly of chickpea transcriptome using short reads for gene discovery and marker identification. DNA Res. 2011;18:53-63.

27. Rogers ME, Colmer TD, Frost K, Henry D, Cornwall D, Hulm E, Deretic J, Hughes SR, Craig AD. Diversity in the genus Melilotus for tolerance to salinity and waterlogging. Plant Soil. 2008;304:89-101. 
28. Stoker JR, Bellis DM. The biosynthesis of coumarin in Melilotus Alba. J Biol Chem. 1962;237:2303-5.

29. Li B, Cong F, Tan CP, Wang SX, Goff SP. Aph2, a protein with a zf-DHHC motif, interacts with c-Abl and has pro-apoptotic activity. J Biol Chem. 2002;277:28870-6.

30. Osterloh JM, Yang J, Rooney TM, Fox AN, Adalbert R, Powell EH, Sheehan AE, Avery MA, Hackett R, Logan MA, MacDonald JM, Ziegenfuss JS, Milde S, Hou Y-J, Nathan C, Ding A, Brown RHJ, Conforti L, Coleman M, Tessier-Lavigne M, Züchner S, Freeman MR. dSarm/Sarm1 is required for activation of an injury-induced axon death pathway. Science. 2012;337:481-4.

31. Maritano D, Sugrue ML, Tininini S, Dewilde S, Strobl B, Fu X, Murray-Tait $V$, Chiarle R, Poli V. The STAT3 isoforms $\alpha$ and $\beta$ have unique and specific functions. Nat Immunol. 2004;5:401-9.

32. Lam BC-H, Sage TL, Bianchi F, Blumwald E. Role of $\mathrm{SH} 3$ domain-containing proteins in clathrin-mediated vesicle trafficking in Arabidopsis. Plant Cell. 2001;13:2499-512.

33. Wu TD, Watanabe CK. GMAP: a genomic mapping and alignment program for mRNA and EST sequences. Bioinformatics. 2005;21:1859-75.

34. Boyle El, Weng S, Gollub J, Jin H, Botstein D, Cherry JM, Sherlock G. GO:TermFinder-open source software for accessing Gene Ontology information and finding significantly enriched Gene Ontology terms associated with a list of genes. Bioinformatics. 2004;20:3710-5.

35. Robinson MD, McCarthy DJ, Smyth GK. edgeR: a Bioconductor package for differential expression analysis of digital gene expression data. Bioinformatics. 2010;26:139-40.

36. Zhao Q-Y, Wang Y, Kong Y-M, Luo D, Li X, Hao P. Optimizing de novo transcriptome assembly from short-read RNA-Seq data: a comparative study. BMC Bioinformatics. 2011;12(S14):2.

37. Daines B, Wang H, Wang L, Li Y, Han Y, Emmert D, Gelbart W, Wang X, Li W, Gibbs R, Chen R. The Drosophila melanogaster transcriptome by paired-end RNA sequencing. Genome Res. 2011;21:315-24.

38. Bahn JH, Lee J-H, Li G, Greer C, Peng G, Xiao X. Accurate identification of A-to-l RNA editing in human by transcriptome sequencing. Genome Res. 2012;22:142-50.

39. Marquez Y, Brown JWS, Simpson C, Barta A, Kalyna M. Transcriptome survey reveals increased complexity of the alternative splicing landscape in Arabidopsis. Genome Res. 2012;22:1184-95.

Ready to submit your research? Choose BMC and benefit from:

- fast, convenient online submission

- thorough peer review by experienced researchers in your field

- rapid publication on acceptance

- support for research data, including large and complex data types

- gold Open Access which fosters wider collaboration and increased citations

- maximum visibility for your research: over 100M website views per year

At $\mathrm{BMC}$, research is always in progress.

Learn more biomedcentral.com/submissions 